\title{
Resveratrol Inhibits Phenotype Modulation by Platelet Derived Growth Factor-bb in Rat Aortic Smooth Muscle Cells
}

\author{
Mi Hee Lee, ${ }^{1}$ Byeong-Ju Kwon, ${ }^{1,2}$ Hyok Jin Seo, ${ }^{1,2}$ Kyeong Eun Yoo, ${ }^{1,2}$ Min Sung Kim, ${ }^{1,2}$ \\ Min-Ah Koo, ${ }^{1,2}$ and Jong-Chul Park ${ }^{1,2}$ \\ ${ }^{1}$ Cellbiocontrol Laboratory, Department of Medical Engineering, Yonsei University College of Medicine, \\ 134 Shinchon-Dong, Seodaemun-Gu, Seoul 120-752, Republic of Korea \\ ${ }^{2}$ Brain Korea 21 PLUS Project for Medical Science, Yonsei University, 134 Shinchon-Dong, Seodaemun-Gu, Seoul 120-752, \\ Republic of Korea \\ Correspondence should be addressed to Jong-Chul Park; parkjc@yuhs.ac
}

Received 8 November 2013; Revised 8 January 2014; Accepted 27 January 2014; Published 10 March 2014

Academic Editor: Constantinos Pantos

Copyright (C) 2014 Mi Hee Lee et al. This is an open access article distributed under the Creative Commons Attribution License, which permits unrestricted use, distribution, and reproduction in any medium, provided the original work is properly cited.

\begin{abstract}
Dedifferentiated vascular smooth muscle cells (VSMCs) are phenotypically modulated from the contractile state to the active synthetic state in the vessel wall. In this study, we investigated the effects of resveratrol on phenotype modulation by dedifferentiation and the intracellular signal transduction pathways of platelet derived growth factor-bb (PDGF-bb) in rat aortic vascular smooth muscle cells (RAOSMCs). Treatment of RAOSMCs with resveratrol showed dose-dependent inhibition of PDGF-bb-stimulated proliferation. Resveratrol treatment inhibited this phenotype change and disassembly of actin filaments and maintained the expression of contractile phenotype-related proteins such as calponin and smooth muscle actin-alpha in comparison with only PDGF-bb stimulated RAOSMC. Although PDGF stimulation elicited strong and detectable Akt and mTOR phosphorylations lasting for several hours, Akt activation was much weaker when PDGF was used with resveratrol. In contrast, resveratrol only slightly inhibited phosphorylations of 42/44 MAPK and p38 MAPK. In conclusion, RAOSMC dedifferentiation, phenotype, and proliferation rate were inhibited by resveratrol via interruption of the balance of Akt, 42/44MAPK, and p38MAPK pathway activation stimulated by PDGF-bb.
\end{abstract}

\section{Introduction}

Vascular smooth muscle cells (VSMC) exhibit differentiated, biosynthetic, contractile roles in the media layer of mature blood vessels. However, VSMC dedifferentiation is induced in response to injury in a vessel, followed by phenotypic modulation toward a proliferative, migratory, and synthetic phenotype with extracellular matrix protein deposition, which contributes to intimal hyperplasia [1-3]. Differentiated VSMCs in normal vessels have a contractile phenotype, a spindle-like elongated morphology, and a smaller cell size. In contrast, dedifferentiated VSMCs in injured vessels have a synthetic phenotype, a hypertrophic appearance, hill and valley growth, and a relatively larger size. In addition to these morphological and functional alterations, the change in VSMCs from contractile to synthetic phenotype is controlled by SMC-specific molecular markers such as caldesmon, calponin, alpha-tropomysin, smooth muscle myosin heavy chain, SM22 $\alpha$, and smooth muscle alpha actin ( $\alpha$ SMA) [4-6].

Platelet derived growth factor-bb (PDGF-bb) is one of the most potent mitogens and chemoattractants for VSMCs. PDGF-bb binds to the PDGF receptor (PDGFR)- $\beta$ and utilizes the tyrosine kinase receptor signaling leading to generation of reactive oxygen species (ROS) and subsequently activates several intracellular signaling cascades, including the extracellular signal-regulated kinase (ERK) and p38 mitogen-activated protein kinase (MAPK) pathways and the phosphatidylinositol 3-kinase-Akt (PI3K-Akt) pathway. It has also been shown to stimulate VSMC dedifferentiation [79]. Akt is the major signal transducer in growth factormediated transcription and promotes cell survival by inhibiting apoptosis. Furthermore, the Akt pathway is the key trigger of mTOR signaling, and Akt-mediated phosphorylation is directly related to mTOR activation. The mTOR protein has 
been implicated in cardiovascular diseases and specifically in cardiac hypertrophy $[10,11]$.

Resveratrol (3,4',5-trihydroxystilbene), a naturally occurring molecule known as a phytoalexin, is a polyphonic compound found in grapes and red wine. Resveratrol is also known to possess antioxidant, anti-inflammatory, antithrombotic, and antiproliferative effects. Additionally, various studies have shown that resveratrol inhibits the oxidation of low-density lipoprotein (oxLDL) and the early progression of atherosclerotic lesions and also protects cardiomyocytes against ischemia-reperfusion injury [12-14]. Although numerous studies have addressed the effects of red wine consumption on cardioprotection, alcohol contained in red wine interacts with resveratrol to elicit the desired effects. For that reason, several studies have demonstrated that both resveratrol supplement and dealcoholized red wine have physiological activity for cardiovascular protection $[15$, 16].

In this study, we investigated the effects of resveratrol on proliferation, phenotype modulation, and intracellular signal transduction pathways in PDGF-bb-induced rat aortic vascular smooth muscle cells (RAOSMCs). Our results demonstrate the inhibitory mechanism of resveratrol on phenotype modulation of PDGF-bb-stimulated RAOSMCs.

\section{Materials and Methods}

2.1. Cell Culture. Primary cultured rat aortic smooth muscle cells (RAOSMCs, Biobud, Seoul, Korea) were routinely maintained in Dulbecco's modified Eagle's medium (DMEM) (Gibco, Carlsbad, CA, USA) supplemented with $10 \%$ fetal bovine serum (Sigma, St. Louis, MO, USA) and a $1 \%$ antibiotic-antimycotic solution containing 10,000 units penicillin, $10 \mathrm{mg}$ streptomycin, and $25 \mu \mathrm{g} / \mathrm{mL}$ amphotericin B (Sigma) at $37^{\circ} \mathrm{C}$ in a humidified atmosphere of $5 \% \mathrm{CO}_{2}$. For experiments, cells were used between passages 5 and 10 .

\subsection{Cell Stimulation by PDGF-bb and Treatment of Resver-} atrol. RAOSMCs were grown to $80-90 \%$ confluence and synchronized in serum-free DMEM medium for $48 \mathrm{~h}$ before experiments. Trans-resveratrol (Sigma) was dissolved in 50\% dimethylsulphoxide (DMSO) (Sigma) for a stock solution of $100 \mathrm{mM}$ and then diluted to desired concentrations with media prior to cell treatment. Cells were treated with various concentrations of resveratrol: $10 \sim 200 \mu \mathrm{M}$ in cell proliferation assay, $20 \mu \mathrm{M}$ in cell morphology analysis, and $100 \mu \mathrm{M}$ in western blotting on quiescent cells with or without $10 \mathrm{ng} / \mathrm{mL}$ PDGF-bb for designated times.

2.3. Cell Proliferation and DNA Synthesis. Cell viability was determined by MTT assay [reduction of 3-(4,5dimethylthiazol-2-yl)-2,5-diphenyltetrazolium bromide to a purple formazan product, Sigma]. For the MTT assay, cells were incubated with $0.5 \mathrm{mg} / \mathrm{mL}$ MTT in the last $4 \mathrm{~h}$ of the culture period and tested at $37^{\circ} \mathrm{C}$ in the dark. The media were decanted, the produced formazan salts were dissolved in DMSO, and the absorbance was determined at $570 \mathrm{~nm}$ by an automatic microplate reader (Spectra Max 340, Molecular
Devices Co., Sunnyvale, CA, USA). DNA synthesis was performed by a 5 -bromo-2' -deoxyuridine (BrdU) incorporation assay (Roche Applied Science, Seoul, Korea). Briefly, BrdUlabeling solution was added to the cells, and cells were incubated for $2 \mathrm{~h}$ at $37^{\circ} \mathrm{C}$. The labeling medium was then removed, and cells were incubated with fixation solution for $30 \mathrm{~min}$ at room temperature. After fixation of the cells, anti-BrdUPOD working solution was added, and cells were incubated for $90 \mathrm{~min}$ at room temperature. Then, the substrate solution was added, and the absorbance was measured at $370 \mathrm{~nm}$ with a $492 \mathrm{~nm}$ reference wavelength by an automatic microplate reader (Spectra Max 340, Molecular Devices Co.).

2.4. Immunofluorescence Assay. Cells were grown on coverslips to $50 \%$ confluence, serum-starved for $48 \mathrm{~h}$, and then stimulated with or without $10 \mathrm{ng} / \mathrm{mL}$ PDGF-bb and 10 or $20 \mu \mathrm{M}$ resveratrol. Stimulated cells were fixed in $10 \%$ formalin solution and permeabilized with $0.5 \%$ Triton $\mathrm{X}$ 100 in phosphate buffer saline (PBS, pH 7.6). Then, cells were blocked with $5 \%$ bovine serum albumin (BSA, Sigma) and incubated with anti-smooth muscle actin- $\alpha$ ( $\alpha$ SMA, Dako North America Inc., CA, USA) and anti-calponin (Santa Cruz Biotechnology Inc., Sana Cruz, CA, USA). Cells were then incubated with the secondary antibody, goatanti-mouse IgG-conjugated Texas Red (Santa Cruz). Alexa 488-conjugated rhodamine phalloidin $(5 \mathrm{U} / \mathrm{mL}$, Invitrogen, Carlsbad, CA, USA) was used to visualize F-actin stress fibers, and nuclei were stained with Hoechst 33528. Coverslips were mounted with aqueous mounting medium (Dako Faramount, Dako North America Inc.), and images were evaluated using fluorescence microscope (Olympus, Tokyo, Japan) equipped with a DP-71 digital camera (Olympus).

2.5. Morphology Analysis. For morphology analysis, a cell plasma membrane was visualized by staining it with Texas Red C2-maleimide (5 U/mL, Invitrogen) and Hoechst 33258 $(1 \mu \mathrm{g} / \mathrm{mL}$ in PBS, Sigma). Images were captured on a fluorescence microscope (Olympus) equipped with a DP-71 digital camera (Olympus). Cell circularity and an area of 100 cells for each group were analyzed using ImageJ software $(\mathrm{NIH}$, Bethesda, MD, USA). The circularity was measured to determine the morphological distribution between the contractile phenotype and the synthetic phenotype of RAOSMCs. Circularity was presented from 0 to 1 , with values closer to 0 indicating spindle morphology and those closer to 1 indicating a circular phenotype $[17,18]$.

2.6. Western Blotting. After time-course stimulation with PDGF-bb, the cells were washed twice with cold PBS $(10 \mathrm{mM}$, $\mathrm{pH}$ 7.4). Ice-cold RIPA lysis buffer (Santa Cruz Biotechnology) was added to the cells for $5 \mathrm{~min}$. The cells were scraped, and the lysate was cleared by centrifugation at $14,000 \times \mathrm{g}$ for $20 \mathrm{~min}$ at $4^{\circ} \mathrm{C}$. The resultant supernatant (total cell lysate) was collected. The protein concentration was determined using a DC Bio-Rad assay kit (Bio-Rad Laboratories, Hercules, California, USA). For immunoblot analysis, proteins were separated by $10-15 \%$ SDS-PAGE and then electrotransferred onto a PVDF membrane. The membrane was blocked with 
blocking buffer ( $5 \%$ bovine serum albumin and 1\% Tween-20 in $20 \mathrm{mM}$ TBS, $\mathrm{pH}$ 7.6) for $1 \mathrm{~h}$ at room temperature and then probed overnight with antibodies to phospho-PDGFR- $\beta$ (p-PDGFR- $\beta$, Tyr751), total PDGFR- $\beta$, phospho-MEK1/2 (pMEK1/2, Ser217/221), total MEK1/2, phospho-p42/44MAPK (p-p42/44MAPK, Thr202/Tyr204), total p42/44MAPK, phospho-Akt (p-Akt, Ser473), total Akt, phospho-mTOR (p-mTOR, Ser2448), total mTOR, phospho-p38 MAPK (pp38MAPK, Thr180/Tyr182), and total p38MAPK. Antibodies were purchased from Cell Signaling Technology (Danvers, MA, USA) and used at 1:1,000 dilutions. Detection of horseradish peroxidase-conjugated secondary antibody (i.e., anti-rabbit $\operatorname{IgG}(1: 2,000)$ and antimouse $\operatorname{IgG}(1: 2,000)$ from Santa Cruz Biotechnology Inc.) was accomplished using enhanced chemiluminescence of the ECL Plus detection kit (Amersham Biosciences, Buckinghamshire, England). The band intensity was quantified using ImageJ software $(\mathrm{NIH})$ and relative fold exchanges averaged across the three experiments (normalized to phosphorylated forms and total forms) are shown below each band.

2.7. Statistical Analysis. All variables were tested in three independent cultures for each experiment. The results are reported as mean $\pm \mathrm{SD}$ compared to nontreated controls. Statistical analysis was performed using a one-way ANOVA, followed by a Tukey's HSD test for multiple comparisons using SPSS software. A $P$ value $<0.05$ was considered statistically significant.

\section{Results}

3.1. Inhibitory Effect of Resveratrol on PDGF-bb-Induced Proliferation in RAOSMCs. To assess whether resveratrol inhibited PDGF-bb-stimulated RAOSMC proliferation, serumstarved RAOSMCs were incubated with $10 \mathrm{ng} / \mathrm{mL}$ PDGF$\mathrm{bb}$ and increasing concentrations of resveratrol for $48 \mathrm{~h}$. Treatment with $10 \mathrm{ng} / \mathrm{mL}$ PDGF-bb induced proliferation of RAOSMCs in comparison with nonstimulated cells. However, the presence of resveratrol resulted in significant $(P<$ 0.05 ) dose-dependent decreases in cell growth (Figure 1(a)). When cells were treated with increasing concentrations of resveratrol, a significant $(P<0.05)$ dose-dependent reduction in cell growth was observed starting at $50 \mu \mathrm{M}$. The level of DNA synthesis was also measured by cell proliferation assay. Stimulation of RAOSMCs with $10 \mathrm{ng} / \mathrm{mL}$ PDGF-bb caused a significant increase in the DNA amount, and resveratrol significantly inhibited this increase in a concentrationdependent manner (Figure 1(b)). Furthermore, increases in cell viability and DNA synthesis induced by PDGF-bb stimulation were completely suppressed in cells treated with concentrations of resveratrol greater than $100 \mu \mathrm{M}$. These results suggest that resveratrol exerts a potent antiproliferative effect on PDGF-bb-stimulated RAOSMC proliferation.

\subsection{Inhibitory Effect of Resveratrol on PDGF-bb-Stimulated} RAOSMC Morphology and Phenotype. To define phenotype exchange in PDGF stimulation, we assessed RAOSMC phenotype and morphology using immunofluorescence and

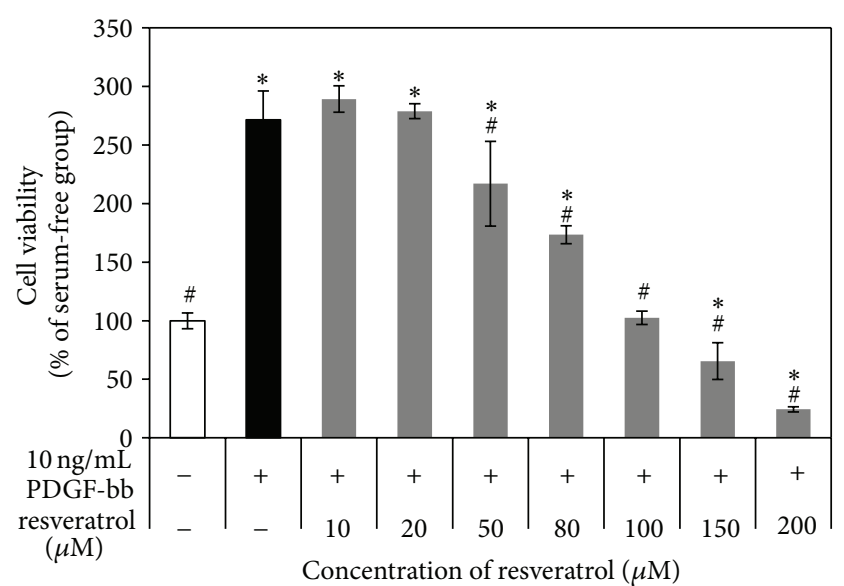

(a)

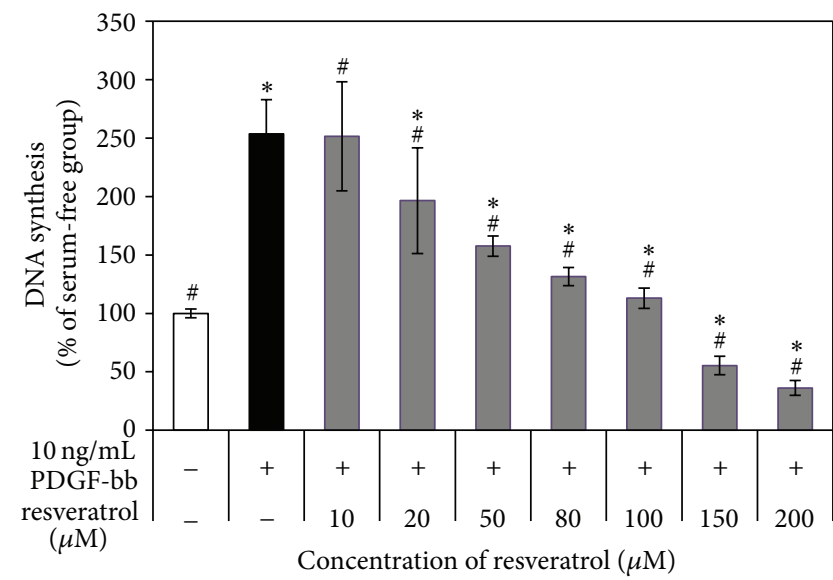

(b)

FIGURE 1: Antiproliferative activity of resveratrol in PDGF-bbstimulated RAOSMCs. After $24 \mathrm{~h}$ of starvation with serum-free DMEM, cells were treated with $10 \mathrm{ng} / \mathrm{mL}$ PDGF-bb and increasing concentrations $(10-200 \mu \mathrm{M})$ of resveratrol for $48 \mathrm{~h}$. (a) The effect of resveratrol growth inhibition on PDGF-bb-stimulated RAOSMCs. Cell viability was detected using the MTT assay. (b) The effect of resveratrol on PDGF-bb-induced DNA synthesis in RAOSMCs. DNA synthesis was detected using the BrdU incorporation assay. ${ }^{*} P<0.05$ compared with nonstimulated controls; ${ }^{\#} P<0.05$ compared with $10 \mathrm{ng} / \mathrm{mL}$ PDGF-bb-stimulated controls.

immunocytochemical staining with antibodies against $\alpha \mathrm{SMA}$ and calponin. RAOSMCs were grown on glass cover slips, starved of serum for $48 \mathrm{~h}$, and stimulated in the presence and absence of PDGF-bb and resveratrol for $24 \mathrm{~h}$.

RAOSMCs exhibited elongation and spindle morphology during prolonged serum deprivation. As shown in Figure 2, RAOSMCs serum-starved for $48 \mathrm{hr}$ revealed an aligned arrangement of actin filaments with an organized cytoskeleton network. In contrast, PDGF-bb-stimulated RAOSMCs showed disassembled distribution and aggregation around the perinuclear region of actin filaments without clear filamentous organization. However, RAOSMCs treated with resveratrol maintained spindle-like shapes and organization of the actin filaments by inhibiting the effects of PDGF-bbstimulation. 

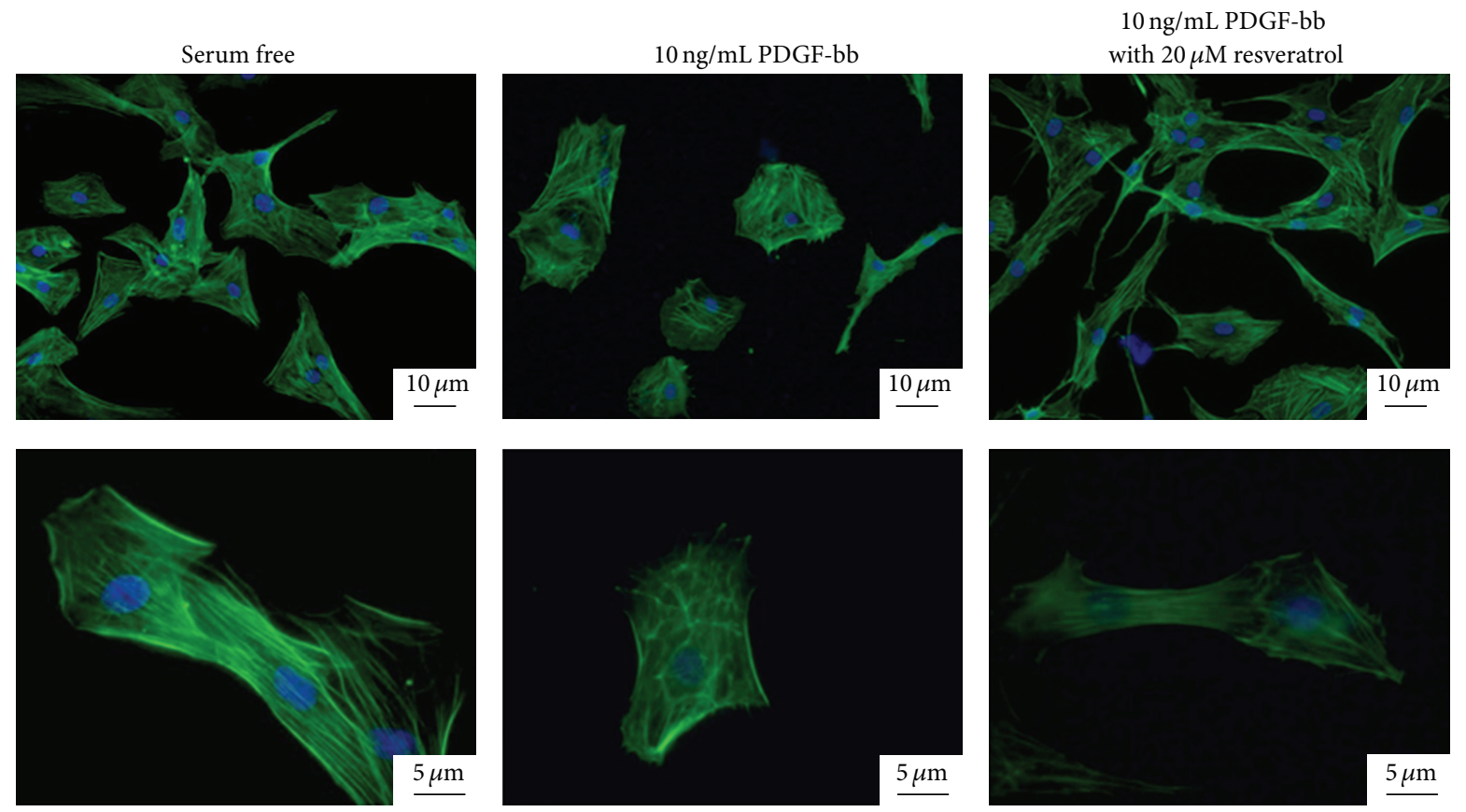

FIGURE 2: Arrangement of F-actin filaments of RAOSMCs with or without $10 \mathrm{ng} / \mathrm{mL}$ PDGF-bb stimulation and $20 \mu \mathrm{M}$ resveratrol. Nuclei were stained blue with Hoechst 33528, and F-actin was green due to alexa (388)-rhodamine phalloidin. Images in the upper and low panels were obtained at $\times 400$ and $\times 1000$ original magnifications, respectively. The micrographs shown in this figure are representative of three independent experiments with similar results.

Therefore, cells were examined for accumulation of the contractile-related proteins, $\alpha$ SMA (Figure $3(\mathrm{a})$ ) and calponin (Figure 3(d)), in clear actin filament organization. When RAOSMCs were stimulated with PDGF-bb, cells were observed to undergo morphological changes from spindleshaped to polygonal, and relatively low levels of $\alpha \mathrm{SMA}$ (Figure 3(b)) and calponin were noted (Figure 3(e)) with disassembled distribution of actin filaments in the cytosol. However, treatment with $20 \mu \mathrm{M}$ resveratrol inhibited the morphological changes. Furthermore, when cells were treated with resveratrol, the actin cytoskeleton maintained parallel actin filaments by forming a complex with contractile phenotyperelated proteins, including $\alpha$ SMA (Figure $3(\mathrm{c})$ ) and calponin (Figure 3(f)). To determine the morphological distribution of the cells, circularity was measured by ImageJ analysis. Compared to serum-starved cells, PDGF-bb-stimulated cells showed a greater distribution in circularity, whereas cells treated with resveratrol exhibited lower circularity (Figure 4(a)). The average circularity of PDGF-bb-stimulated cells was significantly higher than that of nontreated cells. However, resveratrol-treated cells stimulated with PDGFbb were not significantly different from the nontreated cells (Figure 4(b)). Therefore, PDGF-bb-stimulated RAOSMCs had a greater area compared with serum-starved RAOSMCs. Resveratrol inhibited the change in area stimulated by PDGFbb (Figure 4(c)).

3.3. Inhibitory Mechanism of Dedifferentiation on PDGFbb-Stimulated RAOSMCs by Resveratrol. Treatment of
RAOSMCs with resveratrol significantly inhibited PDGFbb-stimulated proliferation in a dose-dependent manner. Furthermore, treatment of cells with $100 \mu \mathrm{M}$ resveratrol almost completely inhibited the growth of ROASMCs, as shown by DNA synthesis assay and MTT assay.

To define the effects of resveratrol on signaling pathways involved in PDGF-bb-stimulated dedifferentiation, serumstarved cells were stimulated with $10 \mathrm{ng} / \mathrm{mL}$ PDGF-bb in the absence or presence of resveratrol for specified times. Then, we analyzed the activation of p42/44MAPK, p38MAPK, and Akt, downstream effectors of PDGF-bb-induced signaling, by Western blotting.

Addition of $10 \mathrm{ng} / \mathrm{mL}$ PDGF-bb to serum-starved cells led to PDGFR- $\beta$ phosphorylation that peaked within $10 \mathrm{~min}$ of stimulation and returnedto baseline level after $2 \sim 4 \mathrm{~h}$, with similar results achieved in atleast three independent experiments (Figure 5(a)). However, resveratrol treatment inhibited PDGFR- $\beta$ phosphorylation induced byPDGF-bb at only $10 \mathrm{~min}$, while no inhibition of PDGFR- $\beta$ phosphorylation was observed during incubations longer than $30 \mathrm{~min}$.

In a similar manner, PDGF-bb stimulated the phosphorylations of downstream effectors such as MEK1/2, p42/44MAPK (Figure 5(b)), and p38 (Figure 5(c)), and resveratrol only slightly inhibited the PDGF-bb-induced phosphorylations of MEK1/2, p42/44MAPK, and p38MAPK in a time-dependent manner. However, as shown in Figure 5(d), while PDGF stimulation elicited strong and detectable signals for Akt and mTOR phosphorylations for several hours, resveratrol treatment clearly inhibited the 


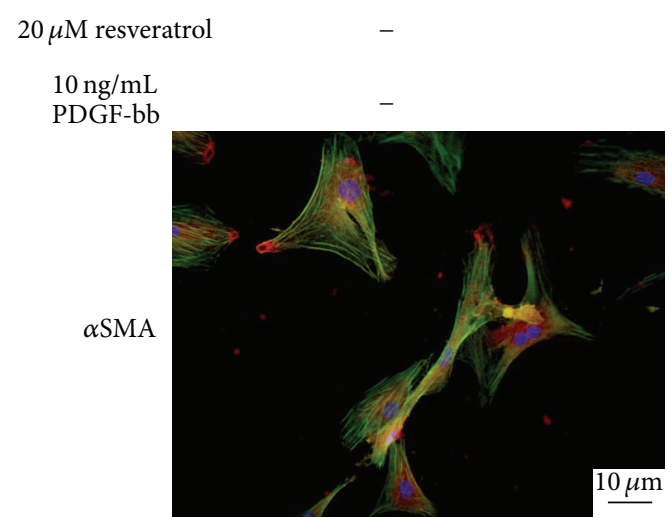

(a)

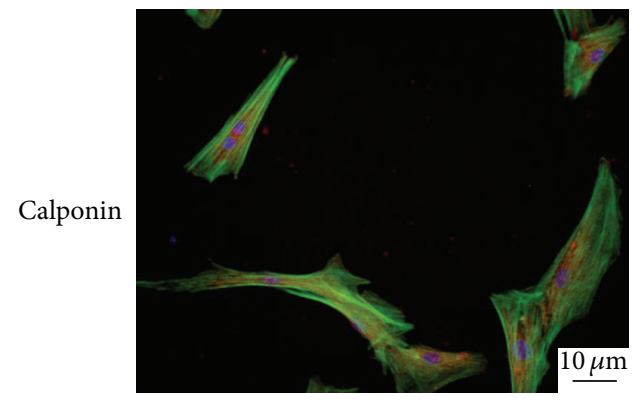

(d)

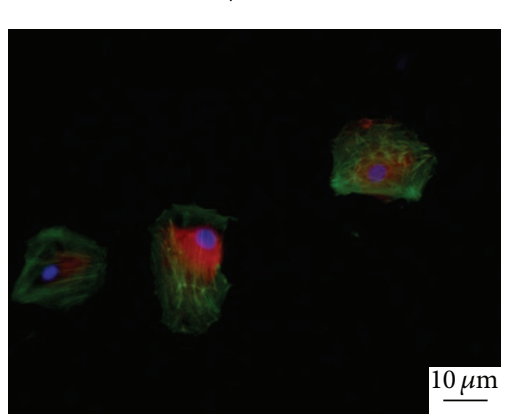

(b)

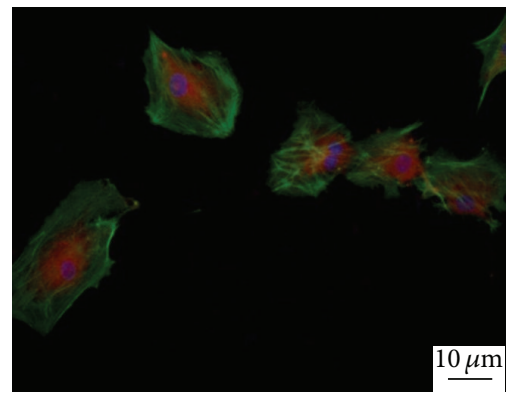

(e)
$+$

$+$

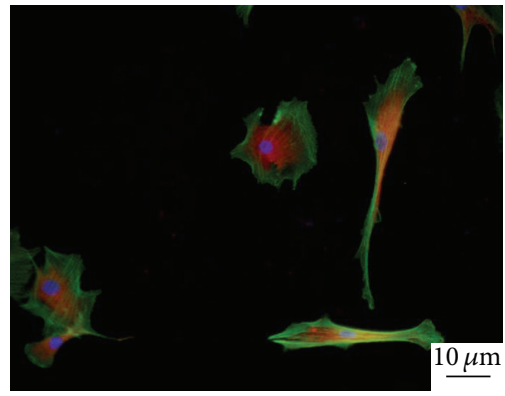

(c)

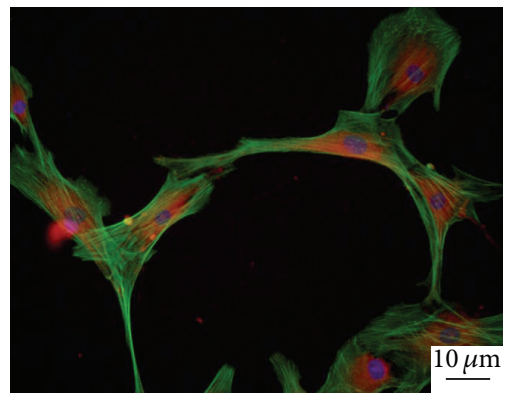

(f)

FIGURE 3: Characterization of morphology modulation by resveratrol on PDGF-bb-stimulated RAOSMCs. Cells were incubated in serum-free media (a, d), $10 \mathrm{ng} / \mathrm{mL}$ PDGF-bb (b, e), or $20 \mu \mathrm{M}$ resveratrol with $10 \mathrm{ng} / \mathrm{mL}$ PDGF-bb (c, f). Nuclei were stained blue with Hoechst 33528 , $\alpha$ SMA $(\mathrm{a} \sim \mathrm{c}$ ) and calponin $(\mathrm{d} \sim \mathrm{f})$ are red, and F-actin is green due to alexa (388)-rhodamine phalloidin. The micrographs (magnification, $\times 100)$ shown in this figure are representative of three independent experiments with similar results.

PDGF-mediated phosphorylations of Akt and mTOR, a downstream effector dependent on Akt.

\section{Discussion}

Changes of the differentiated VSMC play a critical role in cardiovascular diseases, such as atherosclerosis, hypertension, asthma, and vascular aneurisms. Identification of dedifferentiated VSMCs was based on morphological criteria that met the terms for "phenotypic modulation" or "phenotypic switching" in functional and structural properties. A phenotypic switch from a contractile to synthetic phenotype accompanies the proliferation and migration of cells $[19,20]$.

PDGF, a key mediator in the proliferation of VSMCs, plays an important role in the pathogenesis of various vascular disorders. It has already been reported that PDGFbb is implicated in intracellular ROS generation and VSMC growth $[7,21]$. Furthermore, the signaling pathways affected by PDGF are so similar to that activated by oxidative stress $[22,23]$. PDGF-bb represses the characteristic VSMC gene expression by activating extracellular signal-regulated kinase 1/2-mitogen activated protein kinase (ERK1/2-MAPK), p38 MAPK, and Akt pathways in cultured VSMCs [24, 25].

This study reports that PDGF-bb treatment causes phenotypic changes typical of dedifferentiation in RAOSMCs by activating p42/44 MAPK, p38 MAPK, and Akt pathways. In our experimental system, resveratrol inhibited the proliferation of PDGF-bb-stimulated RAOSMCs. The contractile morphology and spindle phenotype of the cells were also preserved by resveratrol treatment. Therefore, markers of VSMC differentiation, such as $\alpha$ SMA and calponin, were not inhibited by resveratrol treatment. In other words, resveratrol might prevent morphologic changes from contractile to synthetic phenotype. VSMCs in mature animal vessels exhibit a contractile phenotype (differentiated state) and express multiple contractile proteins, including $\alpha \mathrm{SMA}, \mathrm{SM} 22 \alpha$, and SM-MHC $[26,27]$. Calponin and $\alpha$ SMA are characterized in detail as F-actin binding components of smooth muscle thin filaments, and they control actin-based cellular processes by regulating the stability of the actin cytoskeleton $[6,28]$.

The investigation of signal transduction pathways induced by PDGF-bb showed that resveratrol can inhibit phosphorylation of PDGFR- $\beta, 42 / 44$ MAPK, Akt, and p38MAPK. In particular, Akt/mTOR phosphorylation was preferentially inhibited in PDGF-bb-induced cells treated with resveratrol. It is known that VSMC phenotype is determined by changes in the balance of activation between the Akt pathway, the ERK, and p38MAPK pathways. Therefore, the Akt pathway plays a vital role in maintaining the differentiated phenotype [29]. 


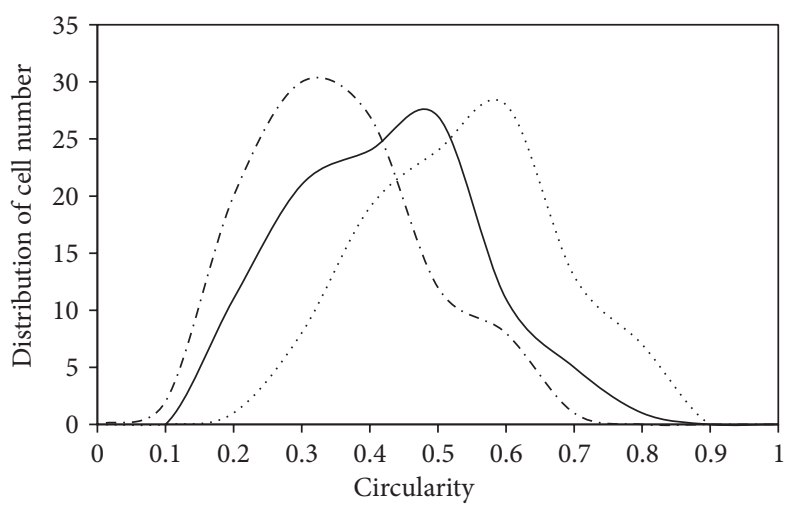

(a)

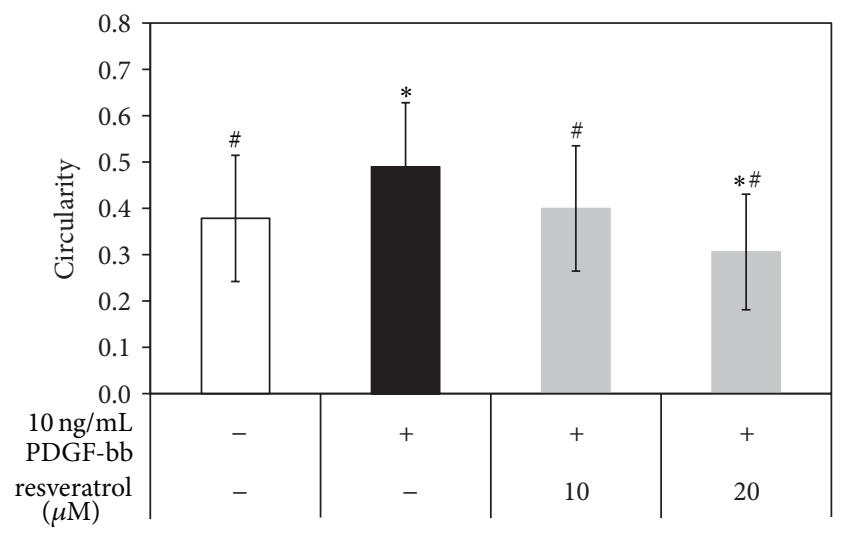

(b)

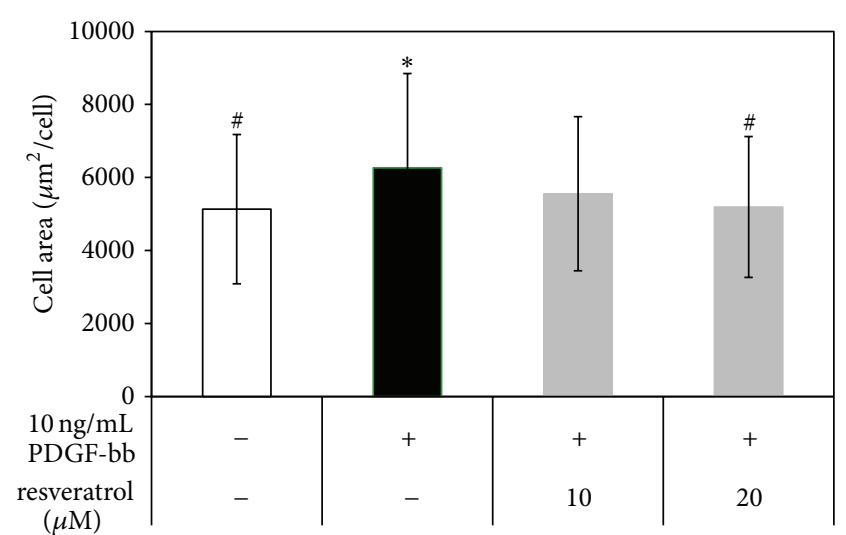

(c)

FIGURE 4: Morphology modulation by resveratrol in PDGF-bb-stimulated RAOSMCs. (a) The distribution of circularity ranged from 0 to 1, for linear to circular, respectively. (—in serum free, —•- in $10 \mathrm{ng} / \mathrm{mL}$ PDGF-bb, and $\bullet . \cdots$ in $10 \mathrm{ng} / \mathrm{mL}$ PDGF-bb with $20 \mu \mathrm{M}$ resveratrol). (b) The average circularity \pm SD was obtained from 100 single cells per each type. ${ }^{*} P<0.05$ compared with nonstimulated control; ${ }^{\#} P<0.05$ compared with $10 \mathrm{ng} / \mathrm{mL}$ PDGF-bb-stimulated control. (c) The average area $\left(\mu \mathrm{m}^{2} /\right.$ cell $) \pm$ SD was obtained from 100 single cells of each type. ${ }^{*} P<0.05$ compared with nonstimulated control; ${ }^{*} P<0.05$ compared with $10 \mathrm{ng} / \mathrm{mL}$ PDGF-bb-stimulated control.

The phosphoinositol-Akt-mammalian target of the rapamycin-p70S6 kinase (PIK/Akt/mTOR/p70S6K) pathway regulates cell growth and cell differentiation in response to nutrients, growth factors, and cytokines [30, 31]. Pharmacological inhibition with rapamycin was shown to induce contractile morphology, SM2-MHC, and calponin, protein reduction, and collagen synthesis in cultures of synthetic phenotype VSMCs by regulation of the mTOR/p70 S6 K1 pathway [30]. Previous studies have shown that mTOR activation induced SMC proliferation and required the activation of the signaling cascade PI3K/PDK1/Akt, as assessed by the effect of the PI3K inhibitors wortmannin and Ly294002, which block PDK $[10,11,32]$. Thus, resveratrol inhibited SMC phenotypic modulation by changing the balance between the Akt and MAPK pathways via hindering PDGF-bb-induced Akt pathways, but not the ERK and p38MAPK pathways. Furthermore, inhibition of Akt/mTOR pathways by resveratrol in SMC affected not only DNA synthesis, but also expression of phenotype-related proteins. Acute or chronic administration of plant polyphenols in patients has been found to result in the vasoprotective, antiangiogenic, antiatherogenic, vasorelaxant, and antihypertensive effects [33]. Several reports demonstrate resveratrol's efficacy in inhibiting VSMC proliferation $[34,35]$. Furthermore, it has been shown that resveratrol specifically blocks the PI3K/PDK1/Akt pathway, thereby inhibiting oxLDL-induced SMC proliferation [14].

In this study, we focused on the effect of resveratrol on phenotypic modulation of RAOSMCs following stimulation with PDGF-bb. From a general point of view, resveratrol exhibits various potentially inhibitory properties on dedifferentiation, including an antiproliferative effect and an ability to modulate important growth signaling pathways.

\section{Conclusion}

In this study, we investigated the effect of resveratrol on dedifferentiation of RAOSMCs induced by PDGF-bb. Resveratrol inhibited dedifferentiation, phenotypic alterations, and proliferation rate stimulated by PDGF-bb in RAOSMC. In conclusion, our results indicate that this effect was probably mediated via a differential regulation of the balance between Akt, 42/44MAPK, and p38MAPK pathway activations stimulated by PDGF-bb at least in part for the effect of resveratrol. This result suggests that resveratrol may be an inhibitor of the 

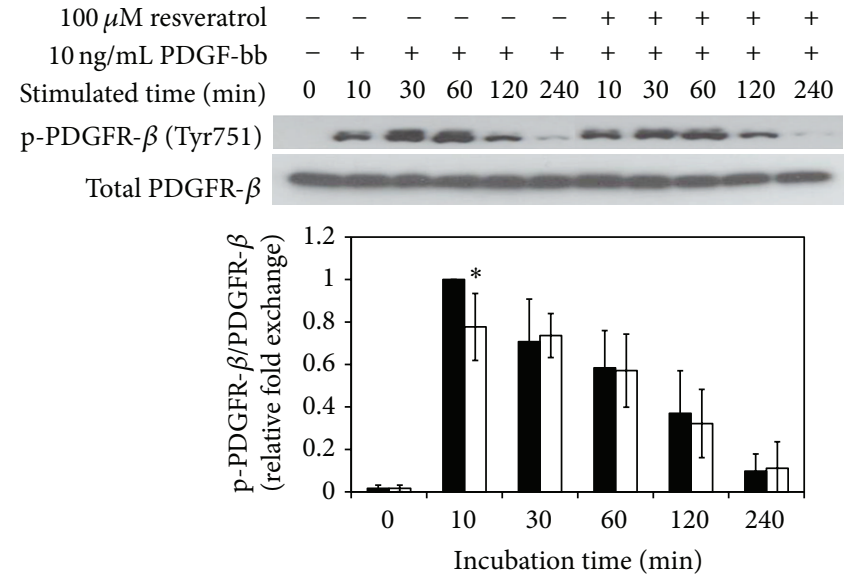

(a)

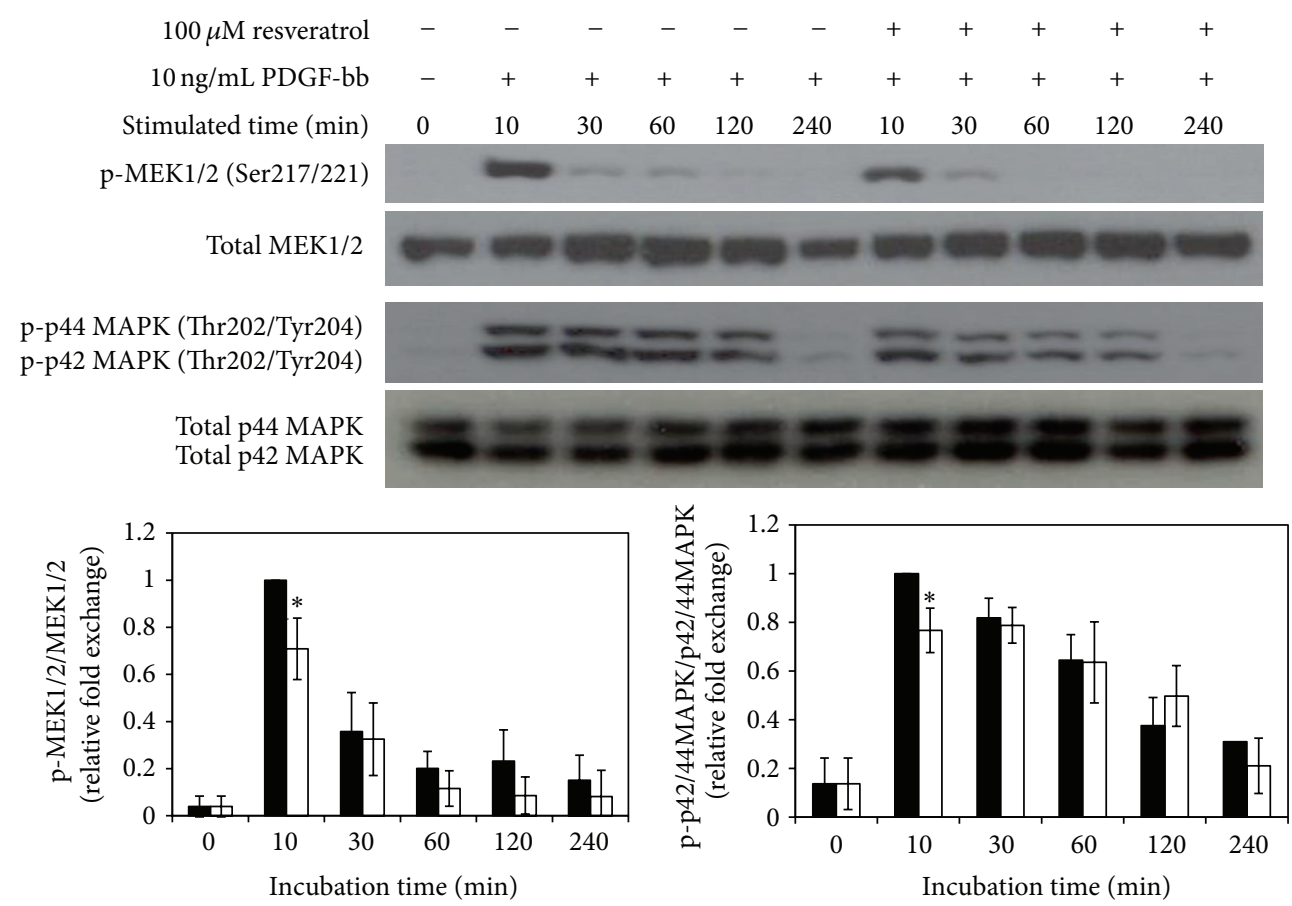

(b)
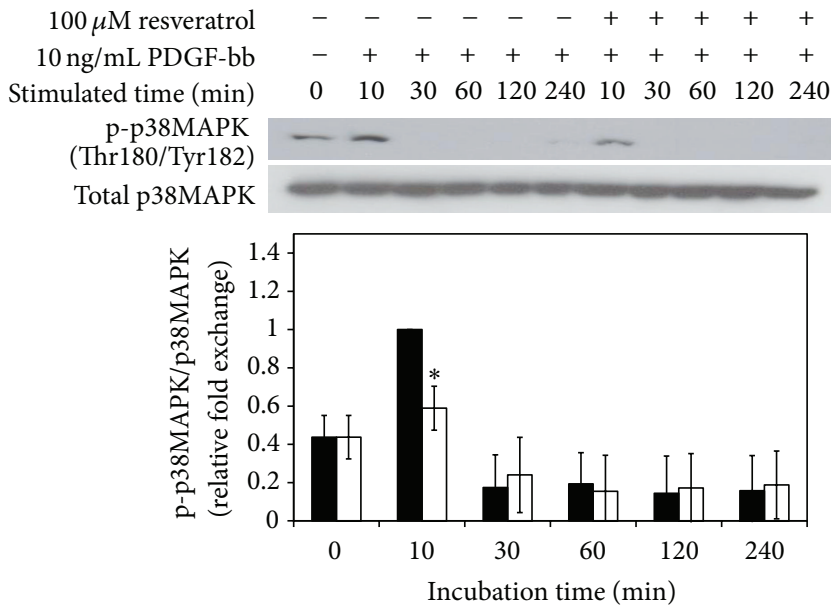

(c)

FIgure 5: Continued. 

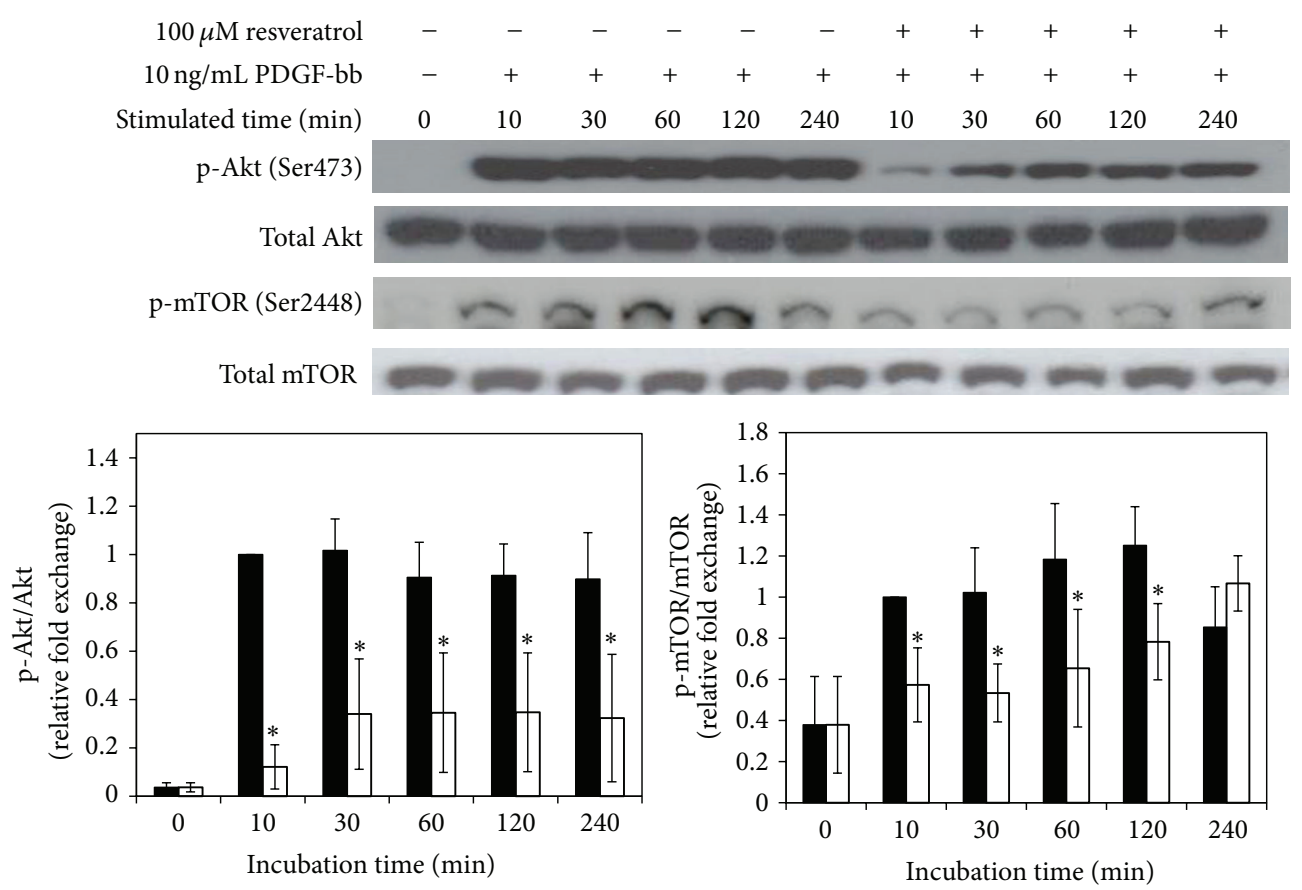

(d)

FIGURE 5: The effects of resveratrol on modulation of PDGF-bb-stimulated signaling pathways in RAOSMCs. RAOSMCs starved of serum were stimulated with $10 \mathrm{ng} / \mathrm{mL}$ PDGF-bb and $100 \mu \mathrm{M}$ resveratrol for the indicated times $(10 \mathrm{~m}, 30 \mathrm{~m}, 1 \mathrm{~h}, 2 \mathrm{~h}$, and $4 \mathrm{~h})$ and lysed. Lysates were immunoblotted with antibodies. After densitometric quantification using the ImageJ program, data were expressed each as the mean \pm SD from three independent experiments. Black bar indicates expression by PDGF-bb stimulation. White bar indicates expression by PDGFbb stimulation with EGCG. (a) The expression of phospho-PDGFR- $\beta$ in a time-dependent manner. The band intensities were normalized to PDGFR- $\beta$ expression. (b) The time-dependent expressions of phospho-MEK1/2 and phospho-p42/44MAPK. The band intensities were normalized to MEK1/2 and p42/44MAPK expression. (c) The time-dependent expression of phospho-p38 MAPK. The band intensities were normalized to p38 MAPK expression. (d) The time-dependent expression of phospho-Akt and phospho-mTOR. The band intensities were normalized to Akt and mTOR expression.

phenotype modulation occurring in arterial stenosis and in postangioplasty restenosis following vascular injury.

\section{Conflict of Interests}

The authors declare that there is no conflict of interests with any financial organization regarding the commercial identities mentioned in the paper.

\section{Acknowledgment}

This study was supported by a grant of the Korea Healthcare Technology R\&D Project, Ministry for Health \& Welfare, Republic of Korea (A120878).

\section{References}

[1] M. G. Davies and P.-O. Hagen, "Pathobiology of intimal hyperplasia," British Journal of Surgery, vol. 81, no. 9, pp. 1254-1269, 1994.

[2] W. D. Coats and D. P. Faxon, "The role of the extracellular matrix in arterial remodelling," Seminars in Interventional Cardiology, vol. 2, no. 3, pp. 167-176, 1997.
[3] S. M. Schwartz, "Smooth muscle migration in atherosclerosis and restenosis," The Journal of Clinical Investigation, vol. 99, no. 12, pp. 2814-2817, 1997.

[4] S. Kenji, H. Ken'ichiro, and N. Wataru, "Molecular mechanism of phenotypic modulation of smooth muscle cells," Hormone Research, vol. 50, supplement 2, pp. 15-24, 1998.

[5] E. M. Rzucidlo, K. A. Martin, R. J. Powell, and N. H. Lebanon, "Regulation of vascular smooth muscle cell differentiation," Journal of Vascular Surgery, vol. 45, no. 6, pp. 25-32, 2007.

[6] G. T. Rozenblum and M. Gimona, "Calponins: adaptable modular regulators of the actin cytoskeleton," International Journal of Biochemistry and Cell Biology, vol. 40, no. 10, pp. 1990-1995, 2008.

[7] M. Sundaresan, Z.-X. Yu, V. J. Ferrans, K. Irani, and T. Finkel, "Requirement for generation of $\mathrm{H}_{2} \mathrm{O}_{2}$ for platelet-derived growth factor signal transduction," Science, vol. 270, no. 5234, pp. 296-299, 1995.

[8] C.-H. Heldin and B. Westermark, "Mechanism of action and in vivo role of platelet-derived growth factor," Physiological Reviews, vol. 79, no. 4, pp. 1283-1316, 1999.

[9] Y. Zhan, S. Kim, Y. Izumi et al., "Role of JNK, p38, and ERK in platelet-derived growth factor-induced vascular proliferation, migration, and gene expression," Arteriosclerosis, Thrombosis, and Vascular Biology, vol. 23, no. 5, pp. 795-801, 2003. 
[10] A. Sekulic, C. C. Hudson, J. L. Homme et al., "A direct linkage between the phosphoinositide 3-kinase-AKT signaling pathway and the mammalian target of rapamycin in mitogen-stimulated and transformed cells," Cancer Research, vol. 60, no. 13, pp. 3504-3513, 2000.

[11] S. Wullschleger, R. Loewith, and M. N. Hall, "TOR signaling in growth and metabolism," Cell, vol. 124, no. 3, pp. 471-484, 2006.

[12] S. Sadruddin and R. Arora, "Resveratrol: biologic and therapeutic implications," Journal of the CardioMetabolic Syndrome, vol. 4, no. 2, pp. 102-106, 2009.

[13] X.-B. Wang, J. Huang, J.-G. Zou et al., "Effects of resveratrol on number and activity of endothelial progenitor cells from human peripheral blood," Clinical and Experimental Pharmacology and Physiology, vol. 34, no. 11, pp. 1109-1115, 2007.

[14] P. M. Brito, R. Devillard, A. Nègre-Salvayre et al., "Resveratrol inhibits the mTOR mitogenic signaling evoked by oxidized LDL in smooth muscle cells," Atherosclerosis, vol. 205, no. 1, pp. 126$134,2009$.

[15] L. Fremont, L. Belguendouz, and S. Delpal, "Antioxidant activity of resveratrol and alcohol-free wine polyphenols related to LDL oxidation and polyunsaturated fatty acids," Life Sciences, vol. 64, no. 26, pp. 2511-2521, 1999.

[16] Z. Wang, J. Zou, K. Cao, T.-C. Hsieh, Y. Huang, and J. M. $\mathrm{Wu}$, "Dealcoholized red wine containing known amounts of resveratrol suppresses atherosclerosis in hypercholesterolemic rabbits without affecting plasma lipid levels," International Journal of Molecular Medicine, vol. 16, no. 1, pp. 533-540, 2005.

[17] H.-J. Sung, S. G. Eskin, Y. Sakurai, A. Yee, N. Kataoka, and L. V. McIntire, "Oxidative stress produced with cell migration increases synthetic phenotype of vascular smooth muscle cells," Annals of Biomedical Engineering, vol. 33, no. 11, pp. 1546-1554, 2005.

[18] S. Li, S. Sims, Y. Jiao, L. H. Chow, and J. G. Pickering, "Evidence from a novel human cell clone that adult vascular smooth muscle cells can convert reversibly between noncontractile and contractile phenotypes," Circulation Research, vol. 85, no. 4, pp. 338-348, 1999.

[19] G. K. Owens, "Regulation of differentiation of vascular smooth muscle cells," Physiological Reviews, vol. 75, no. 3, pp. 487-517, 1995.

[20] G. K. Owens, M. S. Kumar, and B. R. Wamhoff, "Molecular regulation of vascular smooth muscle cell differentiation in development and disease," Physiological Reviews, vol. 84, no. 3, pp. 767-801, 2004.

[21] P. U. Rani, R. Kesavan, R. Ganugula et al., "Ellagic acid inhibits PDGF-BB-induced vascular smooth muscle cell proliferation and prevents atheroma formation in streptozotocin-induced diabetic rats," Journal of Nutritional Biochemistry, vol. 24, no. 11, pp. 1830-1839, 2013.

[22] K. K. Griendling and M. Ushio-Fukai, "Redox control of vascular smooth muscle proliferation," Journal of Laboratory and Clinical Medicine, vol. 132, no. 1, pp. 9-15, 1998.

[23] Y. Taniyama and K. K. Griendling, "Reactive oxygen species in the vasculature: molecular and cellular mechanisms," Hypertension, vol. 42, no. 6, pp. 1075-1081, 2003.

[24] F. Romano, C. Chiarenza, F. Palombi et al., "Platelet-derived growth factor-bb-induced hypertrophy of peritubular smooth muscle cells is mediated by activation of p38 MAP-kinase and of Rho-kinase," Journal of Cellular Physiology, vol. 207, no. 1, pp. 123-131, 2006.

[25] H. P. Reusch, S. Zimmermann, M. Schaefer, M. Paul, and K. Moelling, "Regulation of Raf by Akt controls growth and differentiation in vascular smooth muscle cells," Journal of Biological Chemistry, vol. 276, no. 36, pp. 33630-33637, 2001.

[26] G. K. Owens and G. Wise, "Regulation of differentiation/maturation in vascular smooth muscle cells by hormones and growth factors," Agents and Actions Supplements, vol. 48, pp. 3-24, 1997.

[27] R. S. Blank and G. K. Owens, "Platelet-derived growth factor regulates actin isoform expression and growth state in cultured rat aortic smooth muscle cells," Journal of Cellular Physiology, vol. 142, no. 3, pp. 635-642, 1990.

[28] M. Gimona, I. Kaverina, G. P. Resch, E. Vignal, and G. Burgstaller, "Calponin repeats regulate actin filament stability and formation of podosomes in smooth muscle cells," Molecular Biology of the Cell, vol. 14, no. 6, pp. 2482-2491, 2003.

[29] K. Hayashi, M. Takahashi, W. Nishida et al., "Phenotypic modulation of vascular smooth muscle cells induced by unsaturated lysophosphatidic acids," Circulation Research, vol. 89, no. 3, pp. 251-258, 2001.

[30] M. N. Corradetti and K.-L. Guan, "Upstream of the mammalian target of rapamycin: do all roads pass through mTOR?" Oncogene, vol. 25, no. 48, pp. 6347-6360, 2006.

[31] R. C. Braun-Dullaeus, M. J. Mann, U. Seay et al., "Cell cycle protein expression in vascular smooth muscle cells in vitro and in vivo is regulated through phosphatidylinositol 3-kinase and mammalian target of rapamycin," Arteriosclerosis, Thrombosis, and Vascular Biology, vol. 21, no. 7, pp. 1152-1158, 2001.

[32] N. Auge, V. Garcia, F. Maupas-Schwalm, T. Levade, R. Salvayre, and A. Negre-Salvayre, "Oxidized LDL-induced smooth muscle cell proliferation involves the EGF receptor/PI-3 kinase/Akt and the sphingolipid signaling pathways," Arteriosclerosis, Thrombosis, and Vascular Biology, vol. 22, no. 12, pp. 1990-1995, 2002.

[33] J.-C. Stoclet, T. Chataigneau, M. Ndiaye et al., "Vascular protection by dietary polyphenols," European Journal of Pharmacology, vol. 500, no. 1-3, pp. 299-313, 2004.

[34] A. M. El-Mowafy and R. E. White, "Resveratrol inhibits MAPK activity and nuclear translocation in coronary artery smooth muscle: reversal of endothelin-1 stimulatory effects," FEBS Letters, vol. 451, no. 1, pp. 63-67, 1999.

[35] V. P. Ekshyyan, V. Y. Hebert, A. Khandelwal, and T. R. Dugas, "Resveratrol inhibits rat aortic vascular smooth muscle cell proliferation via estrogen receptor dependent nitric oxide production," Journal of Cardiovascular Pharmacology, vol. 50, no. 1, pp. 83-93, 2007. 


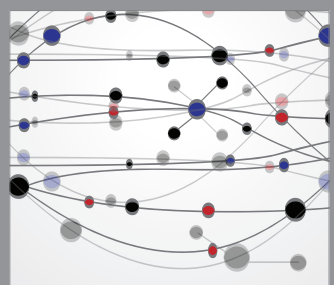

The Scientific World Journal
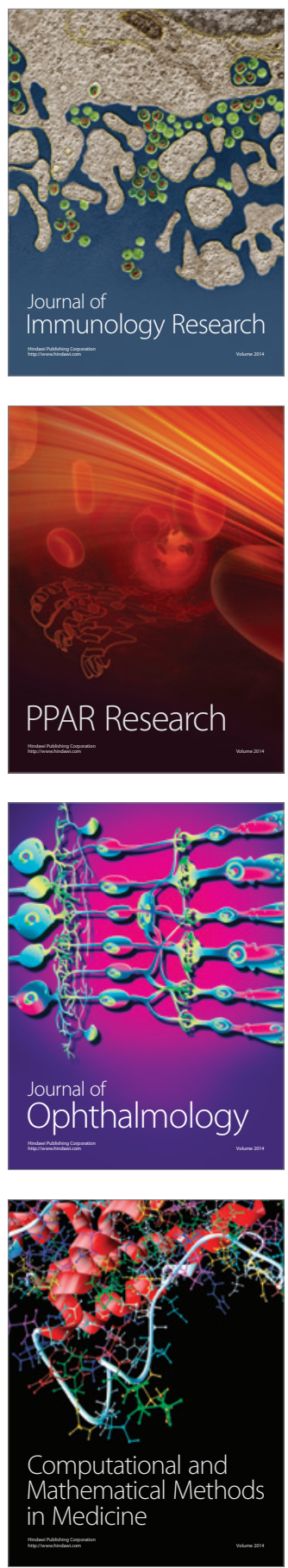

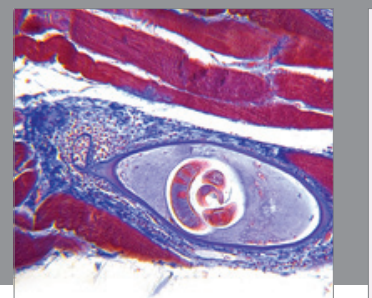

Gastroenterology

Research and Practice
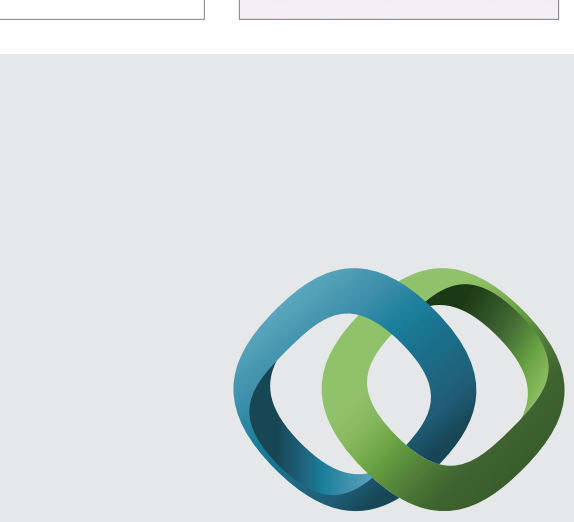

\section{Hindawi}

Submit your manuscripts at

http://www.hindawi.com
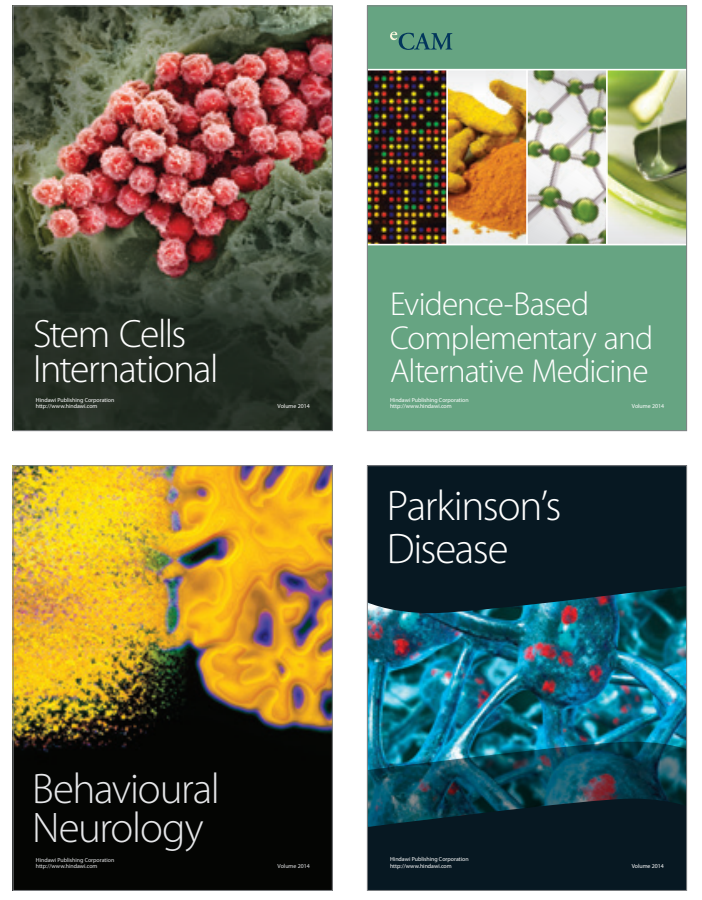
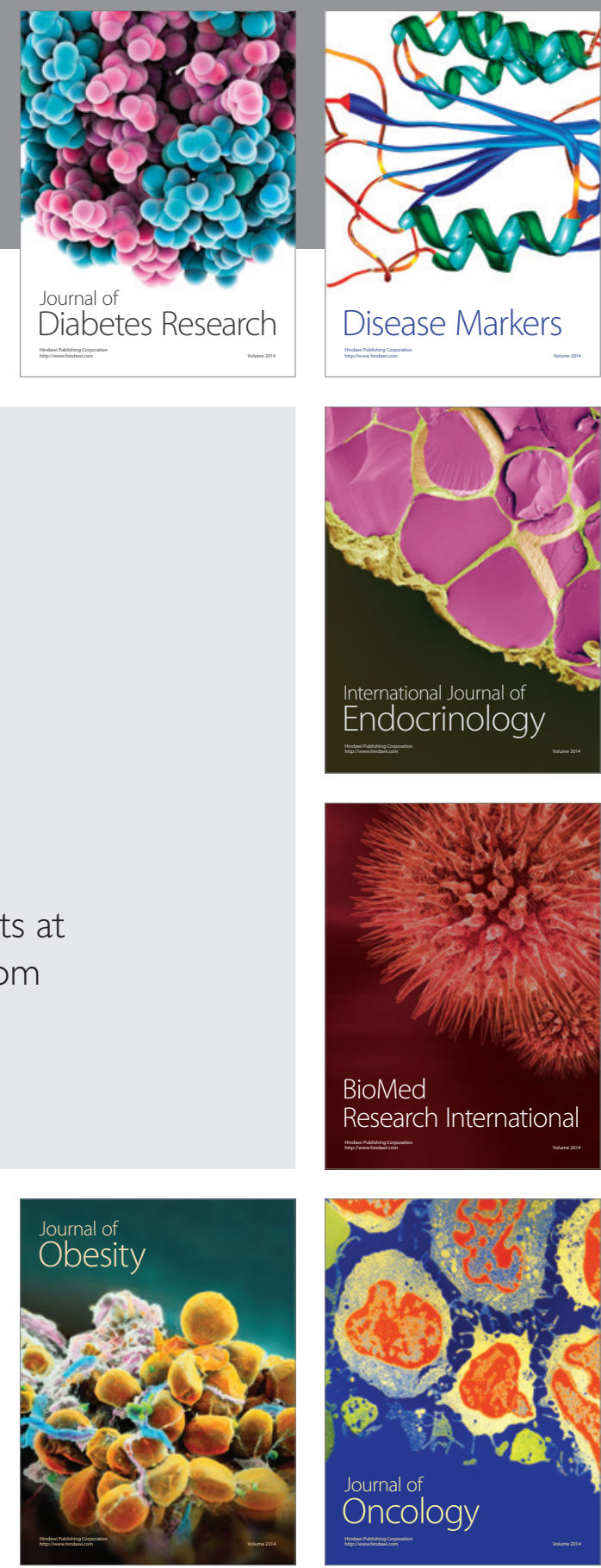

Disease Markers
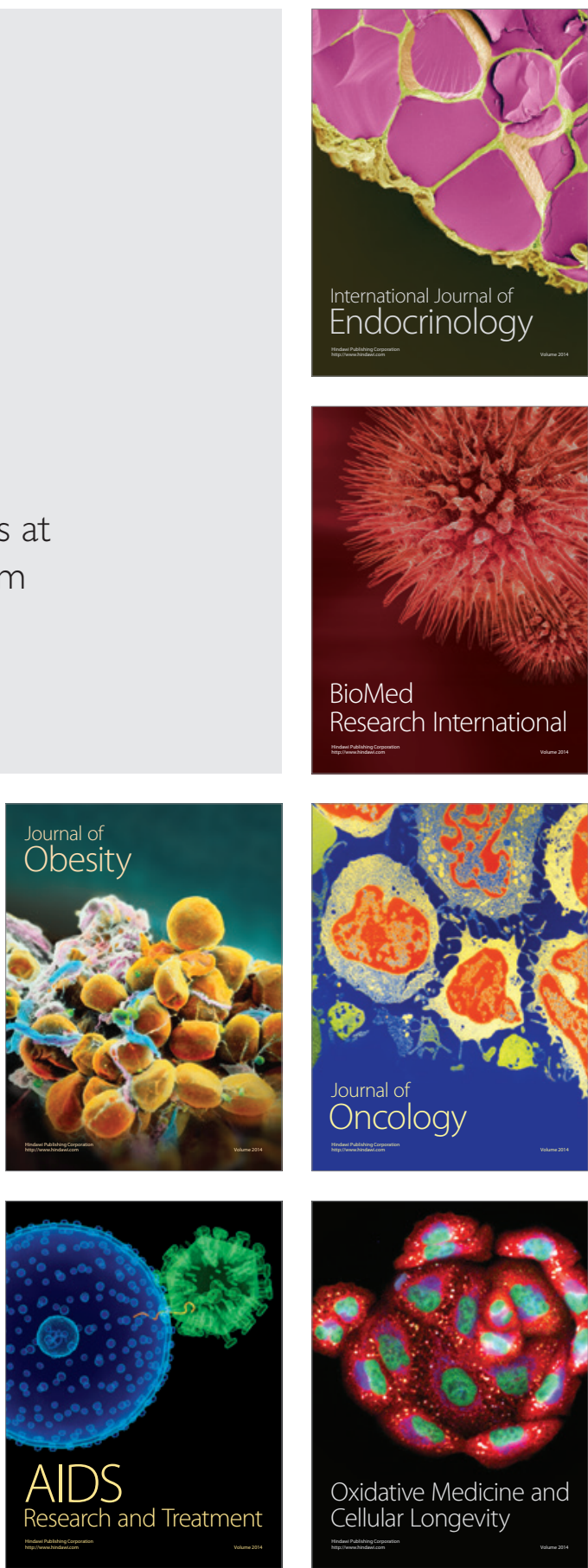